\title{
Study on the influence rule of underlying working face to overlying rock and high level tunnel
}

\author{
Yuan Anying, Zhang Zhenquan, Li Yang \\ (Faculty of Resources and Safety Engineering, China University of Mining and Technology (Beijing ), \\ Beijing 100083,China) \\ yuananying.hi@163.com
}

Keywords: high level tunnel, repeated disruption, stochastic medium theory, instability mechanism

\begin{abstract}
Aiming at the special situation of high level tunnel under the in fluence of mining in Xieqiao coal mine 21116 working face,the theoretical analysis,numerical simulation and field measurement is taken to analyze it.And it reveals the overlying strata movement rule and mechanism of high level tunnel under the in fluence of mining.The results show that the high level east wing orbit large tunnel ids located in bending subsidence belt.With the working face promoting,the movement of overlying strata is a process of forward and upward extension.Controlled by underlying working face mining disturbance again and again,the surrounding rock deformation of high level tunnel is a process of dynamic evolution tension and compression deformation.According to stochastic medium theory,we are carried out on the movement of the overlying rock mass theory parsing.Numerical calculation shows that two parts of the working face displacement form upper and lower respectively showed shape of 'vertical half elliptical' and 'lateral half elliptical'. And a new method is proposed to monitoring the sink of roadway floor that is drilling from earth surface to roadway.This supervise method will provide a new research direction for study of overburden strata movement.In view of the phenomenon of local deformation and failure of roadway,some reinforcement measures must be used to meet the needs of the coal mine safety production.
\end{abstract}

\section{Introduction}

In view of the overlying strata structure and movement law, experts and scholars of domestic and foreign have done a lot of research, and have achieved a lot of results [1-3]. It also makes some research on the working face of the cross mining floor (including longitudinal and cross roadway), Academician Song Zhenqi on the working face of the coal mine and the mine pressure appear on the mountain and the reasonable layout of the roadway is a thorough study, revealing the mine pressure appearance and mechanism of the working face crossing roadway[4].Based on the field measurement and the fuzzy clustering analysis method, Professor Jiang Jinquan has obtained the sub classification model of structural stability of the structure, which can be applied to the stability prediction and support design of the cross mining roadway[5]. Zhu Qinghua established the mechanical model of vertical and cross roadway, and studied the characteristics of surrounding rock stress distribution and the stability of surrounding rock under dynamic pressure influence by using visco elastic plastic theory[6]. Xie Wenbing, et al. use the numerical mechanics analysis method to explain the influence of the dynamic influence of the roadway surrounding rock evolution law and the position of the roadway on the stability of the surrounding rock in the near distance[7-8]. Li Guichen, et al. study the failure characteristics and stability control of surrounding rock in Huaibei mining area, summary the typical characteristics of roadway failure, and put forward the strengthening control technology of surrounding rock[9]. Dai Jin, et al. study the influence of the different working direction on the maintenance of the roadway, through the theory and practice, it is proved that the working surface is greater than the longitudinal span to the stability of the surrounding rock[10].

In conclusion, experts and scholars have done most of the theoretical research but less effective monitoring method of on - site for the overlying strata structure and rock movement, the structure 
and rock movement of cross mining roadway floor was also a part of the study, but the study on the mechanism of the overlying strata in the upper strata in the mining disturbance mechanism is also less. Therefore, the research on the influence mechanism of the mining process of the upper and lower coal face on the deformation and movement of the roadway surrounding rock in the overlying strata, will provide theoretical basis and scientific basis for the research on the movement law of overlying strata, working surface mining design and roadway surrounding rock support design.

In this paper, based on the analysis of the moving law of overlying strata,theoretical calculation, numerical simulation and field monitoring of the comprehensive research method are used to study the surrounding rock movement, damage mechanism, etc, of high roadway caused by lower working surface mining.

\section{Engineering geological conditions}

Xieqiao coal mine of Huainan Mining Group 21116 surface plagioclase is $237.7 \mathrm{~m}$ and the advancing length is $1623 \mathrm{~m}$. The working face of coal seam is not stable, along the trough direction of coal seam floor fluctuation is large, coal seam occurrence is $190^{\circ} \sim 202^{\circ}, \angle 10.8^{\circ} \sim 13.8^{\circ}$. Return air along the slot mining cut eye distance is $623 \mathrm{~m} \sim 1106 \mathrm{~m}$, the thickness of coal seam is $0.8 \mathrm{~m}$ to $2.0 \mathrm{~m}$, mining from open cut eye distance is $1480 \mathrm{~m} \sim 1566 \mathrm{~m}$, the thickness of coal seam is $0.3 \mathrm{~m}$ to $2.5 \mathrm{~m}$. Anterograde transport of slot mining cut eye distance is $541 \mathrm{~m} \sim 849 \mathrm{~m}$, the thickness of coal seam is $1.2 \mathrm{~m} \sim 2.1 \mathrm{~m}$, from the mining cut eye distance is $1250 \mathrm{~m} \sim 1321.5 \mathrm{~m}$, the thickness of coal seam is $0.3 \mathrm{~m} \sim 2.8 \mathrm{~m} .21116$ mining working face is east of the second level in group $\mathrm{B}$ District 6 coal first mining working face, the western boundary is $720 \mathrm{~m}$ East Group B track cross-cut, the eastern boundary is east 11-2 4 coal floor track cross-cut. Groove seam floor elevation is $-701.8 \sim-711.2 \mathrm{~m}$, transport trough floor elevation is $-752.8 \sim-767.8 \mathrm{M}$.

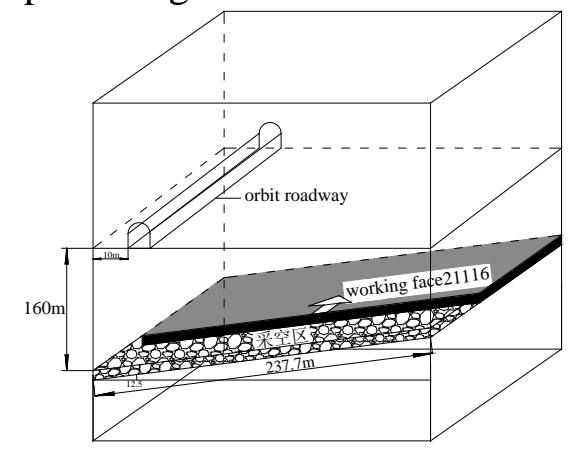

Fig. 1 Working face and roadway space position relationship

In this working face, the $-590 \mathrm{~m}$ level is arranged on the east side of the main entry, the roadway is located in the $10 \mathrm{~m}$, the vertical distance of the roadway is $160 \mathrm{~m}$, and the working face and the space position of the tunnel are shown in Figure 1.

\section{Moving law of overlying strata}

\subsection{Analysis of moving law of overlying strata}

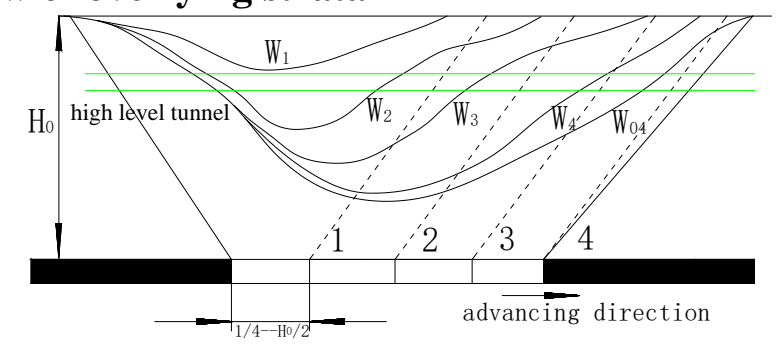

Fig. 2 The overlying strata and surface movement regularity

1,2,3,4-Working surface position ; $\mathrm{W}_{1}, \mathrm{~W}_{2}, \mathrm{~W}_{3}, \mathrm{~W}_{4}$ - On the corresponding working surface of the overlying strata movement ; $\mathrm{W}_{04}$-Final morphology of the overlying strata movement

By the moving law of overlying strata along with the moving direction of the working surface, it 
can be seen that the overlying strata movement is a process of continuous forward and upward extension. It is controlled by repeated disturbance of the working face mining, and the deformation of surrounding rock is a dynamic evolution process, as shown in Figure 2.

\subsection{Rock mass movement theory analysis}

The moving law of the overlying rock mass can be analyzed according to the random medium theory. The displacement and deformation of rock mass caused by excavation is simplified as a plane problem. Because of the 21116 working face in the direction is $237.7 \mathrm{~m}$, the advancing direction will be pushed forward $1617 \mathrm{~m}$, based on the definition of full mining we can know that the moving of the overlying strata will be stable with the working face advancing enough distance. The overlying strata will not continue to advance along with the working surface. It is called "strike full exploitation"; and the direction of the working face length is shorter. If the adjacent working face is bound to cause the overlying strata in the inclined direction to move, it is called "the tendency to not fully exploit", based on the above theory, an analytical model is established, which is shown in Figure 3 which is based on the assumption that the vertical displacement of rock mass is calculated by the(s, z).

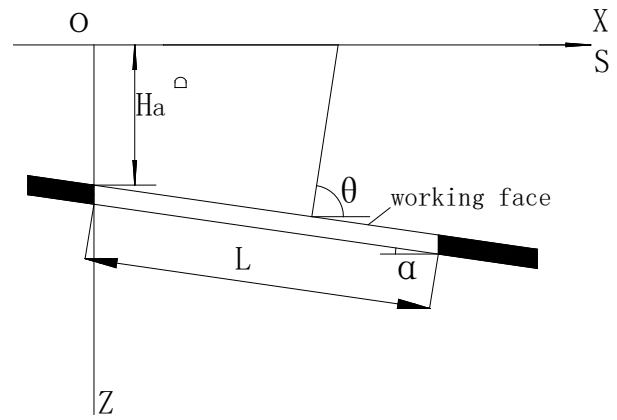

Fig. 3 Analysis model of vertical displacement of rock mass

$$
\begin{aligned}
& W_{e}(x, z)=\frac{\tan \beta(z)}{H_{a}-z+s \tan \alpha} \exp \\
& \left\{\frac{-\tan ^{2} \beta(z)}{\left(H_{a}-z+s \tan \alpha\right)^{2}}\left[x-s-\left(H_{a}-z+s \tan \alpha\right) \cos \theta\right]^{2}\right\}
\end{aligned}
$$

In the formula: $\mathrm{H}_{\mathrm{a}}$ - minimum mining depth, $\alpha$ - seam dip angle, $\mathrm{m}$ - coal seam mining thickness, $\theta$-mining influence angle.

Due to the direction of the working face to the direction is full exploitation, and this working surface is currently a single mining, then the vertical displacement $\mathrm{W}_{(\mathrm{x}, \mathrm{z})}$ of overlying rock caused by 21116 single working surface mining by type (1) can be obtained:

$$
\begin{aligned}
& W(x, z)=W_{\max } \tan \beta(z) \int_{0}^{L \cos \alpha} \frac{1}{H_{a}-z+s \tan \alpha} \\
& \exp \left\{-\frac{\pi \tan ^{2} \beta(z)}{\left(H_{a}-z+s \tan \alpha\right)^{2}}\left[x-s-\left(H_{a}-z+s \tan \alpha\right) \cos \theta\right]^{2}\right\} d s
\end{aligned}
$$

In the formula: L—working face length; $\beta(\mathrm{z})$ — depth of the main influence range of the $\mathrm{Z}$ level; $\eta$-the full exploitation of the sinking coefficient; $k$ - exploitation of the impact of the transmission coefficient; $\mathrm{W}_{\max }$-maximum possible sink,

$$
W_{\max }=\frac{m \eta}{2 \cos [\alpha(1-k)]}
$$

According to the above formula, we can work out the moving value of the rock mass in the upper part of the rock mass after the mining influence.

\section{Analysis on the influence of high level roadway}

\subsection{Numerical simulation scheme}

The model is based on the geological conditions of 21116 working face in the coal mine, and the 3D model of FLAC3D is established. The model goes to 580m, $460 \mathrm{~m}, 380 \mathrm{~m}$, and 459000 cells. 


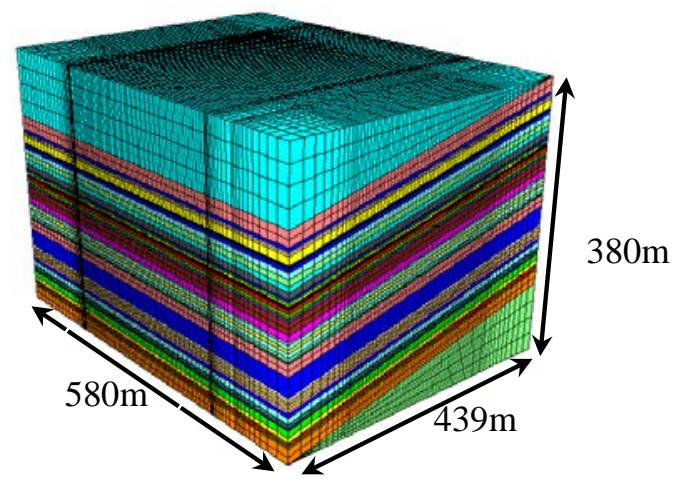

Fig. 4 The sketch of three dimension model

The model limits the lateral movement and the vertical movement of the bottom surface. The weight of the overlying strata is simulated by vertical load, and the strain softening model is selected. The numerical model is shown in Figure 4. Numerical simulation steps: (1) the model of the initial state of the mechanical and displacement conditions at the given boundary conditions; (2) the upper part of the upper part of the excavation; (3) the upper and lower groove; (4) along the direction of the excavation of the 21116 working face.

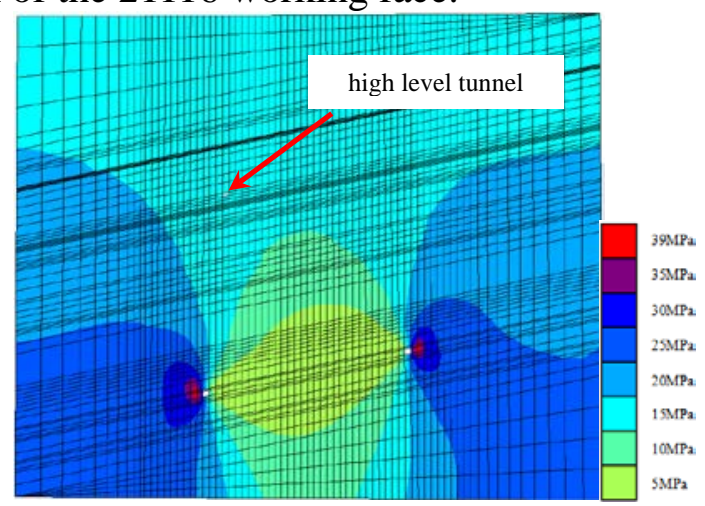

Fig. 5 Stress field image of stope

\subsection{Stress field distribution of high level roadway affected by mining}

According to figure 5, it can be seen that, with the mining of the working face, the upper part of the whole tunnel is in the low stress area, which shows that the stress of the working face is limited to the local influence of the roadway, but only according to the different advancing step of the tunnel caused certain disturbance.

\subsection{Displacement field distribution of high level roadway affected by mining}

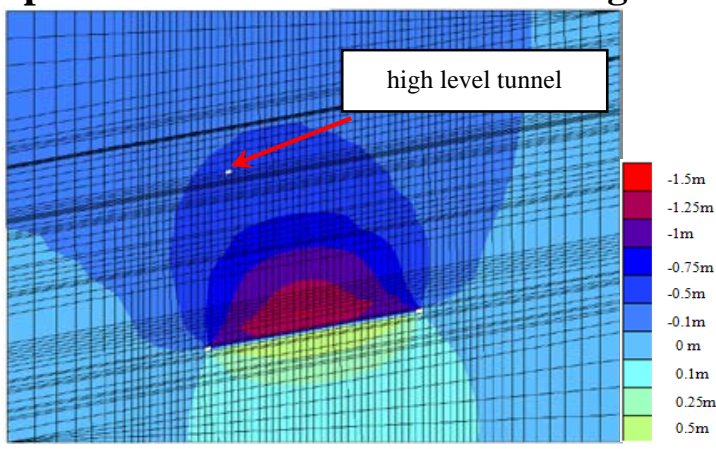

Fig. 6 The orientation of the working face and the displacement of the roadway

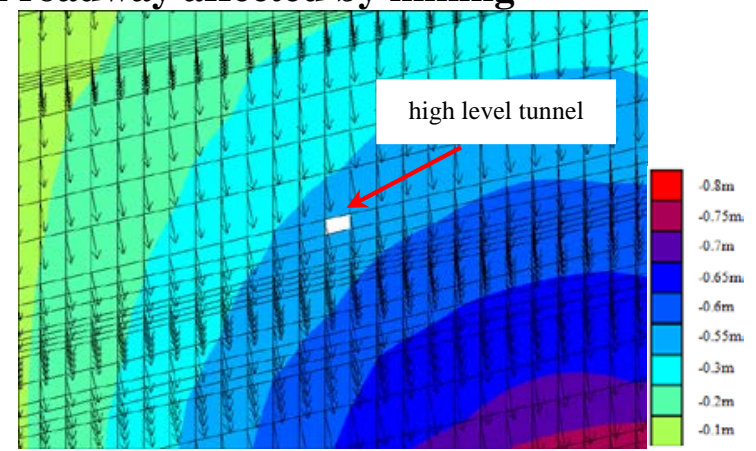

Fig. 7 Displacement vector of surrounding rock of tunnel

From the displacement map of the mining field in the figure 6 we can see that the moving range of the overlying strata in the mining field is "funnel", and the upper part of the working face is larger, and the upper part is lower, the lower part of the mined out area is formed a half ellipse. By the displacement vector map of the roadway in the figure 7 we can see that the displacement direction of the roadway roof and bottom plate are all facing the goaf, and the upper and lower working surface of the mining area is the overall subsidence of the overlying strata. The final subsidence of the roadway is the comprehensive effect of the floor heave and the whole subsidence. 
The overall deformation of the roadway is a trend of subsidence, and the final settlement amount is about $600 \mathrm{~mm}$.

\section{Mechanism analysis of roadway failure}

\subsection{Mechanism analysis of roadway failure}

During the mining process of the lower working face, due to the section of the working surface of the roof subsidence, the roadway is subjected to repeated stretching and compression deformation, the stability of the roadway is under the pressure deformation after the working face mining ${ }^{[11-13]}$. In addition, in the process of coal mining, the dynamic changes of the roadway mainly experienced two processes: (1) the total subsidence caused by the coal mining under the working surface of the lower coal mining; (2) the deformation of the roadway in the process of the tunnel subsidence.

\subsection{Analysis of roadway deformation law}

(1) Analysis of roadway failure process

From the field of roadway damage analysis, in the distance from the open cut horizontal distance $117.3 \mathrm{~m}$ position of the pulp began to fall off, in the $202-287 \mathrm{~m}$ range of large pulp skin off, $130-135 \mathrm{~m}$ range of two $\mathrm{U}$ type shed roof fracture, and the $1.5 \mathrm{~m}$ radius of the water phenomenon, $162.3 \mathrm{~m}$ position along the direction of the axial direction of the crack, and in the entire affected by the working surface, other parts of the region also appeared in the pulp and water. This is due to the long axis of the object to bear the deformation of the capacity is far less than the short axis, the direction of the long axis of the roadway is more prone to deformation, and because the direction of the direction of the roadway direction and direction, the direction of the roof and floor of the roadway in a certain angle, the surrounding rock will produce lateral tension and long-term support to the pressure of the bearing pressure along with the advancing gradually stabilized. Under the dynamic influence of the vertical horizontal tension and lateral support pressure, the surface of the tunnel is segmented, and the skin is loose and the local water is poured out.

(2) Monitoring and analysis of the subsidence of the roadway floor

In this paper in order to get the floor of the east wing rail tunnel subsidence, innovation put forward from the surface play vertical drilling to the east wing rail tunnel method, selected surface of a fixed point as the basis, the observation line along the East flank orbit drift towards the direction of arrangement of, monitoring scope of $1750 \mathrm{~m}$. Coexistence of floor roadway layout 18 measuring points, such as measured data, figure 2 shown (Figure 8 shows subsidence are all after the completion of the working face mining roadway floor final subsidence)



Fig. 8 The sinkage of the roadway floor

Monitoring results show that, due to the occurrence of coal seam in the coal seam, the maximum of the roadway floor subsidence is the largest, close to $700 \mathrm{~mm}$, and the rest of the roadway has a small fluctuation, and the average subsidence is about $550 \mathrm{~mm}$. The working face to promote $1617 \mathrm{~m}$, according to the working face mining on the overlying strata of the lead, the impact on the high level of the range of $1716 \mathrm{~m}$, including the roadway floor subsidence in the range of $1400 \mathrm{~m}$ $550 \mathrm{~mm}-700 \mathrm{~mm}$ is a large area of roadway.

The monitoring of the damage form and the subsidence quantity of the roadway can be obtained. The high level roadway has a certain deformation or failure condition, but the overall situation 
remains stable, and the stability of the surrounding rock is ensured by using some local reinforcement measures.

\section{Main conclusions}

(1) In this paper, the maximum height of the caving zone is $13.7 \mathrm{~m}$, the maximum height of the fracture zone is $75 \mathrm{~m}$, and the upper part of the East Wing track is located in the upper part of the bend. With the advancing of the working surface, the overlying strata movement is a process of continuous forward and upward extension. The deformation of surrounding rock is a dynamic evolution process controlled by repeated disturbance of the working face mining.

(2) The numerical results show that the upper part of the stope is "upper semi ellipse" and gradually decreased. The lower part of the production area is formed in the lower part of the short axis. The displacement direction is toward the goaf, and the final settlement of the roadway floor is caused by the comprehensive action of the floor heave and the whole subsidence.

(3) This paper proposed and implemented a new method to measure the floor subsidence, which provides a new direction for the field test of the overlying strata movement, and the final measurement of the roadway floor is about $700 \mathrm{~mm}$, and the average is about $550 \mathrm{~mm}$.

(4)During the process of mining, the surrounding rock has experienced a whole subsidence and deformation of the two processes. Although there are some areas of the pulp and water loss, but the overall stability of the roadway, to take a certain strengthening measures to meet the requirements of coal mine safety production.

\section{Acknowledgements}

This Project is supported by the National Natural Science Foundation of China (Grant Nos. 51504005, 51304007), and the open fund laboratory of An Hui University of Science \&technology(Grant No. KLDCMERDPC13104)

Author:Yuan Anying,(1986.09-),He Ze Shangong province.Doctor, China University of Mining and Technology (Beijing ).

\section{References}

[1]Qian Ming-gao,Liu Ting-cheng.Mining pressure and strata control [M].Xu Zhou:China University of Mining and Technology Press,2003.

[2]Xu Jia-lin,Qian Ming-gao.the research on surface movement under the influence of key stratum of strata movement[J].International Journal of coal Science \& Technology,2002,25(2):122-126.

[3]Deng Ka-zhong.The rock mass structure effects of mining subsidence[M].Xu Zhou:China University of Mining and Technology Press,1998.

[4]Song Zhen-qi,Jiang Jin-quan,Liu Yi-xue.Mine pressure appear across alleys and up the hill and the reasonable layout of roadway[J].Coal Seicence and Technology,1987,05:2-5+60-61.

[5]Jiang Jin-quan,Feng Zeng-qiang,Han Ji-sheng.Across the mining structure stability of roadway surrounding rock classification and its application[J].Journal of Engineering Geology,1999,04:321-326.

[6]Zhu Qing-hua.Mechanics analysis of surrounding rock deformation of roadway affected by deep riding mining and its stability control[D]China University of Mining and Technology,2010.

[7]Xie Wen-bing,Shi Zhen-fan,Yin Shao-ju.Stability analysis of surrounding rock masses of roadway under overhead mining[J].Chinese Journal of Rock Mechanics and Engineering,2004,12:1986-1991.

[8]Xie Wen-bing,Shi Zhen-fan,Chen Xiao-xiang,Lu Shi-liang.Stability analyses of roadway surrounding rock induced by overhead mining[J].Journal of China University of Mining \& 
Technology,2004,01:85-88.

[9]LI Gui-chen,Ma Zhen-qian,ZHang Nong,Wang Peng-peng,Ma Rui.Research on failure characteristics and control measures of roadways affected by multiple overhead mining in Huaibei mining area.[J].Journal of Mining and Safety Engineering,2013,02:181-187.

[10]Dai Jin,Li Hong,Li Feng-he,Liu Tong-xin.The influence of face advance direction of mining roadway maintenance[J].Ground Pressure and Strata Control,1999,Z1:190-193.

[11]Guo Qi-lin,Qiao Shi-fan,Liu Bao-chen.The Movement and Deformation of Soil and Rock Mass Resulted from Mining Activity.Journal of Mining and Safety Engineering,2011,01:109-114.

[12]Qiao S F, Liu B C.Prediction of ground displacement and deformation induced by dewatering[C]//ZHU He-hua,ZHANG Feng.Underground Construction and Ground Movement.New York:ASCE,2006:73-79.

[13]Meng Wu-feng,Zhao Zhong-ming,Wu Jian-bang,Wang Geng-yao,Yang Zhi-liang.Study on underlying seam mining affected to upward mine transportation roadway[J].Coal Seicence and Technology,2011,03:6-10. 